\title{
Stability of the second order partial differential equations
}

\author{
M Eshaghi Gordji ${ }^{1,2,3}$, YJ Cho ${ }^{4^{*}}$, MB Ghaemi ${ }^{5}$ and B Alizadeh ${ }^{6,7}$
}

\footnotetext{
* Correspondence: yjcho@gnu.ac.kr ${ }^{4}$ Department of Mathematics Education and the Rins, Gyeongsang National University, Chinju 660-701, Korea

Full list of author information is available at the end of the article
}

$$
\begin{aligned}
& \text { Abstract } \\
& \text { We say that a functional equation }(\xi) \text { is stable if any function } g \text { satisfying the } \\
& \text { functional equation }(\xi) \text { approximately is near to a true solution of }(\xi) \text {. } \\
& \text { In this paper, by using Banach's contraction principle, we prove the stability of } \\
& \text { nonlinear partial differential equations of the following forms: } \\
& \qquad \begin{array}{l}
y_{x}(x, t)=f(x, t, y(x, t)) \text {, } \\
a y_{x}(x, t)+b y_{t}(x, t)=f(x, t, y(x, t)) \\
p(x, t) y_{x t}(x, t)+q(x, t) y_{t}(x, t)+p_{t}(x, t) y_{x}(x, t)-p_{x}(x, t) y_{t}(x, t)=f(x, t, y(x, t)) \\
p(x, t) y_{x x}(x, t)+q(x, t) y_{x}(x, t)=f(x, t, y(x, t))
\end{array}
\end{aligned}
$$

2000 Mathematics Subject Classification. 26D10; 34K20; 39B52; 39B82; $46 B 99$.

Keywords: generalized Hyers-Ulam stability, linear differential equation, Banach's contraction principle

\section{Introduction}

Let $X$ be a normed space over a scalar field $\mathbb{K}$, and let $I$ be an open interval. Assume that, for any function $f: I \rightarrow X(y=f(x))$ satisfying the differential inequality

$$
\left\|a_{n}(t) \gamma^{(n)}(t)+a_{n-1}(t) y^{(n-1)}(t)+\cdots+a_{1}(t) y^{\prime}(t)+a_{0}(t) y(t)+h(t)\right\| \leq \varepsilon
$$

for all $t \in I$, where $\varepsilon \geq 0$, there exists a function $f_{0}: I \rightarrow X$ satisfying

$$
\left\{\begin{array}{l}
y_{0}=f_{0}(x), \\
a_{n}(t) y_{0}^{(n)}(t)+a_{n-1}(t) y_{0}^{(n-1)}(t)+\cdots+a_{1}(t) y_{0}^{\prime}(t)+a_{0}(t) y_{0}(t)+h(t)=0
\end{array}\right.
$$

and $\left\|f(t)-f_{0}(t)\right\| \leq K(\varepsilon)$ for any $t \in I$.

Then we say that the above differential equation has the Hyers-Ulam stability. If the above statement is also true, then we replace $\varepsilon$ and $K(\varepsilon)$ by $\phi(t)$ and $\varphi(t)$, where $\phi, \varphi: I$ $\rightarrow[0, \infty)$ are functions not depending on $f$ and $f_{0}$ explicitly, then we say that the corresponding differential equation has the Hyers-Ulam-Rassias stability or the generalized Hyers-Ulam stability.

In 1998, the Hyers-Ulam stability of differential equation $y^{\prime}=y$ was first investigated by Alsina and Ger [1]. In 2002, this result has been generalized by Takahasi et al. [2] for the Banach space-valued differential equation $y^{\prime}=\lambda y$. In 2005, Jung [3] proved the generalized Hyers-Ulam stability of a linear differential equation of the first order. For 
more results on stability of differential equations, see also [4-7] and [8] and, for more details on the Hyers-Ulam stability and related topics, the readers refer to [9-17] and [18-20].

In this paper, we prove the Hyers-Ulam-Rassias stability of the following partial differential equations:

(1) The first order nonlinear partial differential equation:

$$
y_{x}(x, t)=f(x, t, y(x, t))
$$

(2) The first order nonlinear partial differential equation:

$$
a y_{x}(x, t)+b y_{t}(x, t)=f(x, t, y(x, t))
$$

for all $a, b \in \mathbb{R}$;

(3) The second order nonlinear partial differential equation:

$$
p(x, t) \gamma_{x x}(x, t)+q(x, t) \gamma_{x}(x, t)=f(x, t, y(x, t))
$$

under the following condition:

$$
p_{x x}(x, t)=q_{x}(x, t) .
$$

The differential equation (1.1) is the second order nonlinear partial differential equation, and we call it exact if the condition (1.2) holds.

(4) The mixed type second order nonlinear partial differential equation:

$$
p(x, t) y_{x t}(x, t)+q(x, t) y_{t}(x, t)+p_{t}(x, t) y_{x}(x, t)-p_{x}(x, t) y_{t}(x, t)=f(x, t, y(x, t))
$$

under the following condition:

$$
p_{x t}(x, t)=q_{t}(x, t) .
$$

Theorem 1.1. (Banach's Contraction Principle) Let $(X, d)$ be a complete matric space and $T: X \rightarrow X$ be a contraction, that is, there exists $\alpha \in[0,1)$ such that

$$
\mathrm{d}(T x, T y) \leq \alpha \mathrm{d}(x, y)
$$

for all $x, y \in X$. Then, there exists $a$ unique $a \in X$ such that $T a=a$. Moreover, $a=$ $\lim _{n \rightarrow \infty} T^{n} x$ and

$$
\mathrm{d}(a, x) \leq \frac{1}{1-\alpha} \mathrm{d}(x, T x)
$$

for all $x \in X$.

\section{Main results}

In this section, let $I=[a, b]$ be a closed interval with $a<b$ and $C(I \times I)=\{f: I \times I \rightarrow$ $\mathbb{R}: f$ is continuous\}. For the sake of convenience, assume that all the integrals and all the derivatives exist. 
Theorem 2.1. Let $c \in I \phi: I \times I \rightarrow(0, \infty)$ be a continuous function, $L: I \times I \rightarrow[1, \infty)$ be an integrable function and $K: I \times I \times \mathbb{R} \rightarrow \mathbb{R}$ be a continuous function. Assume that there exists $0<\beta<1$ such that

$$
\begin{gathered}
\qquad \int_{c}^{x} L(\tau, t) \varphi(\tau, t) \mathrm{d} \tau<\beta \varphi(x, t) ; \\
|K(x, t, u(x, t))-K(x, t, v(x, t))| \leq L(x, t)|u(x, t)-v(x, t)| \\
\text { for all } x, t \in I \text { and } u, v \in C(I \times I) \text {. Let } y: I \times I \rightarrow \mathbb{R} \text { be such that } \\
\qquad\left|y_{x}(x, t)-K(x, t, y(x, t))\right| \leq \varphi(x, t)
\end{gathered}
$$

for all $x, t L I$. Then, there exists a unique continuously differentiable function $y_{0}: I \times$ $I \rightarrow \mathbb{R}$ such that

$$
y_{0}(x, t)=y(c, t)+\int_{c}^{x} K\left(\tau, t, y_{0}(\tau, t)\right) \mathrm{d} \tau
$$

(consequently, $y_{0}$ is a solution to $\left.y_{x}(x, t)=K(x, t, y(x, t))\right)$ and

$$
\left|\gamma(x, t)-y_{0}(x, t)\right| \leq \frac{\beta}{1-\beta} \varphi(x, t)
$$

for all $x, t L I$.

Proof. Let $X$ be the set of all continuously differentiable functions $u: I \times I \rightarrow \mathbb{R}$. We define a metric $d$ and an operator $T$ on $X$ as follows, respectively:

$$
\mathrm{d}(u, v)=\sup _{x, t \in I} \frac{|u(x, t)-v(x, t)|}{\varphi(x, t)}
$$

and the operator

$$
\text { (Tu) }(x, t)=y(c, t)+\int_{c}^{x} K(\tau, t, u(\tau, t)) \mathrm{d} \tau
$$

for all $u \in X$. Using (2.1) and (2.2), we have

$$
\begin{aligned}
\mathrm{d}(T u, T v) & =\sup _{x, t \in I} \frac{\left|\int_{c}^{x}[K(\tau, t, u(\tau, t))-K(\tau, t, v(\tau, t))] \mathrm{d} \tau\right|}{\varphi(x, t)} \\
& \leq \sup _{x, t \in I} \frac{\int_{c}^{x} L(\tau, t)|u(\tau, t)-v(\tau, t)| \mathrm{d} \tau}{\varphi(x, t)} \\
& =\sup _{x, t \in I}\left[\frac{\int_{c}^{x} L(\tau, t) \varphi(\tau, t) \frac{|u(\tau, t)-v(\tau, t)|}{\varphi(\tau, t)} \mathrm{d} \tau}{\varphi(x, t)}\right] \\
& \leq \sup _{x, t \in I} \frac{\int_{c}^{x} L(\tau, t) \varphi(\tau, t) \sup _{\tau, t \in I} \frac{|u(\tau, t)-v(\tau, t)|}{\varphi(\tau, t)} \mathrm{d} \tau}{\varphi(x, t)} \\
& =\mathrm{d}(u, v) \sup _{x, t \in I} \frac{\int_{c}^{x} L(\tau, t) \varphi(\tau, t) \mathrm{d} \tau}{\varphi(\tau, t)} \\
& \leq \beta \mathrm{d}(u, v) .
\end{aligned}
$$


Now, by Theorem 1.1, there exists a unique $y_{0} \in X$ such that $T y_{0}=y_{0}$, that is,

$$
y_{0}(x, t)=\gamma(c, t)+\int_{c}^{x} K\left(\tau, t, y_{0}(\tau, t)\right) \mathrm{d} \tau .
$$

Moreover, by Theorem 1.1, we have

$$
\mathrm{d}\left(y_{0}, y\right) \leq \frac{1}{1-\beta} \mathrm{d}(y, T y)
$$

for all $y \in X$. It follows from (2.3) that

$$
-\varphi(x, t) \leq y_{x}(x, t)-K(x, t, y(x, t)) \leq \varphi(x, t)
$$

for all $x, t \in I$. If we integrate each term in the above inequality from $c$ to $x$, then we get

$$
\begin{aligned}
\mid y(x, t)-\left(y(c, t)-\int_{c}^{x} K(\tau, t, y(\tau, t)) \mathrm{d} \tau \mid\right. & \leq \int_{c}^{x} \varphi(\tau, t) \mathrm{d} \tau \\
& \leq \int_{c}^{x} L(\tau, t) \varphi(\tau, t) \mathrm{d} \tau \\
& \leq \beta \varphi(x, t) .
\end{aligned}
$$

Now, we have

$$
\frac{|y(x, t)-(T y)(x, t)|}{\varphi(x, t)} \leq \beta \Rightarrow \sup _{x, t \in I} \frac{|y(x, t)-(T y)(x, t)|}{\varphi(x, t)} \leq \beta .
$$

Thus, we get

$$
\mathrm{d}(\gamma, T y) \leq \beta
$$

Therefore, by (2.4) and (2.5), we see that

$$
\left|y(x, t)-y_{0}(x, t)\right| \leq \frac{\beta}{1-\beta} \varphi(x, t)
$$

for all $x, t \in I$. This completes the proof. $\square$

Theorem 2.2. Let $c \in I, p, q: I \times I \rightarrow \mathbb{R}$ be continuous functions with $p(x, t) \neq 0$ for all $x, t \in I, \phi: I \times I \rightarrow(0, \infty)$ be a continuous function, $L: I \times I \rightarrow[1, \infty)$ be an integrable function, and $f: I \times I \times \mathbb{R} \rightarrow \mathbb{R}$ be a continuous function. Assume that there exists $0<\beta<1$ such that

$$
\begin{gathered}
\int_{c}^{x} L(\tau, t) \varphi(\tau, t) \mathrm{d} \tau \leq \beta \varphi(x, t) ; \\
h(c, t)=-\left[p(c, t) y_{x}(c, t)-p_{x}(c, t) y(c, t)+q(c, t) y(c, t)\right] ; \\
K(x, t, y(x, t)) \\
=-(p(x, t))^{-1}\left[\left(p_{x}(x, t)-q(x, t)\right) y(x, t)+h(c, t)-\int_{c}^{x} f(\tau, t, \gamma(\tau, t) \mathrm{d} \tau]\right.
\end{gathered}
$$


and

$$
|K(x, t, u(x, t))-K(x, t, v(x, t))| \leq L(x, t)|u(x, t)-v(x, t)|
$$

for all $c, x, t \in I$ and $h, u, v, y \in C(I \times I)$. Let $y: I \times I \rightarrow \mathbb{R}$ be a function such that:

$$
\left|p(x, t) y_{x x}(x, t)+q(x, t) y_{x}(x, t)-f(x, t, y(x, t))\right| \leq \varphi(x, t)
$$

for all $x, t \in I$ and (1.2) holds. Then, there exists a unique solution $y_{0}: I \times I \rightarrow \mathbb{R}$ of (1.1) such that

$$
\left|y(x, t)-y_{0}(x, t)\right| \leq \frac{\beta}{1-\beta} \varphi(x, t) .
$$

Proof. It follows from (1.2) and (2.7) that

$$
\begin{aligned}
& \left|p(x, t) y_{x x}(x, t)+q(x, t) y_{x}(x, t)-f(x, t, y(x, t))\right| \\
= & \mid\left(p(x, t) y_{x}(x, t)-p_{x}(x, t) y(x, t)\right)_{x}+(q(x, t) y(x, t))_{x} \\
& +\left[p_{x x}(x, t)-q_{x}(x, t)\right] y(x, t)-f(x, t, y(x, t)) \mid \\
= & \left|\left(p(x, t) y_{x x}(x, t)-p_{x}(x, t) y(x, t)\right)_{x}+(q(x, t) y(x, t))_{x}-f(x, t, y(x, t))\right| \\
\leq & \varphi(x, t) .
\end{aligned}
$$

Thus, we have

$$
\begin{aligned}
& -\varphi(x, t) \\
\leq & \left(p(x, t) y_{x x}(x, t)-p_{x}(x, t) y(x, t)\right)_{x}+(q(x, t) y(x, t))_{x}-f(x, t, y(x, t)) \\
\leq & \varphi(x, t) .
\end{aligned}
$$

By using (2.8), we get

$$
\begin{aligned}
& \left|p(x, t) y_{x}(x, t)-p_{x}(x, t) y(x, t)+q(x, t) y(x, t)+h(c, t)-\int_{c}^{x} f(\tau, t, y(\tau, t)) \mathrm{d} \tau\right| \\
= & |p(x, t)| \mid \gamma_{x}(x, t)+(p(x, t))^{-1}\left(\left(q(x, t)-p_{x}(x, t)\right) \gamma(x, t)+h(c, t)\right. \\
& \left.-\int_{c}^{x} f(\tau, t, y(\tau, t)) \mathrm{d} \tau\right) \mid \\
\leq & \int_{c}^{x} \varphi(\tau, t) \mathrm{d} \tau,
\end{aligned}
$$

where

$$
h(c, t)=-\left[p(c, t) \gamma_{x}(c, t)-p_{x}(c, t) y(c, t)+q(c, t) y(c, t)\right] .
$$

From (2.9), it follows that

$$
\begin{aligned}
& \left|y_{x}(x, t)+(p(x, t))^{-1}\left(\left(q(x, t)-p_{x}(x, t)\right) y(x, t)+h(c, t)-\int_{c}^{x} f(\tau, t, \gamma(\tau, t)) \mathrm{d} \tau\right)\right| \\
\leq & |p(x, t)|^{-1} \int_{c}^{x} \varphi(\tau, t) \mathrm{d} \tau .
\end{aligned}
$$


From $p(x, t)=\frac{p(x, t)\left[1+(p(x, t))^{2}\right]}{1+(p(x, t))^{2}}$, without less of generality, we can assume that $|p(x, t)|$ $\geq 1$.

Now, By putting

$$
\begin{aligned}
& K(x, t, y(x, t)) \\
= & -(p(x, t))^{-1}\left[\left(p_{x}(x, t)-q(x, t)\right) y(x, t)+h(c, t)-\int_{c}^{x} f(\tau, t, y(\tau, t) \mathrm{d} \tau]\right.
\end{aligned}
$$

in the above inequality, we get

$$
\begin{aligned}
\left|y_{x}(x, t)-K(x, t, y(x, t))\right| & \leq|p(x, t)|^{-1} \int_{c}^{x} \varphi(\tau, t) \mathrm{d} \tau \\
& \leq \int_{c}^{x} \varphi(\tau, t) \mathrm{d} \tau \\
& \leq \int_{c}^{x} L(\tau, t) \varphi(\tau, t) \mathrm{d} \tau \\
& \leq \beta \varphi(x, t) \\
& \leq \varphi(x, t) .
\end{aligned}
$$

Thus, the conclusions of the Theorem follows from Theorem 2.1. This completes the proof. $\square$

If (1.1) is multiplied by a function $\mu(x, t)$ such that the resulting equation is exact, that is,

$$
\mu(x, t)\left[p(x, t) y_{x x}(x, t)+q(x, t) y_{x}-f(x, t, y(x, t))\right]=0
$$

and

$$
(\mu(x, t) p(x, t))_{x x}-(q(x, t) \mu(x, t))_{x}=0,
$$

then we say that $\mu(x, t)$ is an integrating factor of the partial differential equation (1.1).

Corollary 2.3. Let $p, q, \mu: I \times I \rightarrow \mathbb{R}$ be continuous functions such that $p(x, t) \neq 0$ and $\mu(x, t) \neq 0$ for all $x, t \in I$, and (2.10) holds. Assume that $c \in I, L: I \times I \rightarrow[1, \infty)$ is an integrable function and $f: I \times I \times \mathbb{R} \rightarrow \mathbb{R}$ is a continuous function. Suppose that there exists $0<\beta<1$ such that

$$
\begin{gathered}
\int_{c}^{x} L(x, t) \varphi(\tau, t) \mathrm{d} \tau \leq \beta \varphi(x, t) ; \\
h(c, t)=-\left[\mu(c, t) p(x, t) y_{x}(c, t)-(\mu p)_{x}(c, t) y(c, t)+\mu(c, t) q(c, t) y(c, t)\right] \\
K(x, t, \gamma(x, t))=-(\mu(x, t) p(x, t))^{-1}\left[\left(\mu(x, t) q(x, t)-(\mu q)_{x}(x, t)\right) y(x, t)\right. \\
\left.+\left(h(c, t)-\int_{c}^{x} \mu(\tau, t) f(\tau, t, \gamma(\tau, t)) \mathrm{d} \tau\right)\right]
\end{gathered}
$$


and

$$
|K(x, t, u(x, t))-K(x, t, v(x, t))| \leq L(x, t)|u(x, t)-V(x, t)| .
$$

for all c, $x, t \in I$ and $h, u, v \in C(I \times I)$. Let $y: I \times I \rightarrow \mathbb{R}$ be a function such that

$$
|\mu(x, t)|\left|p(x, t) y_{x x}(x, t)+q(x, t) y_{x}(x, t)-f(x, t, y(x, t))\right| \leq \varphi(x, t)
$$

for all $x, t \in I$ and the condition (2.11) holds. Then, There exists a unique solution $y_{0}$ $: I \times I \rightarrow \mathbb{R}$ of (2.10) such that

$$
\left|\gamma(x, t)-y_{0}(x, t)\right| \leq \frac{\beta}{1-\beta} \varphi(x, t) .
$$

Proof. It follows from Theorem 2.2 that

$$
y_{0}(x, t)=\gamma(c, t)+\int_{c}^{x} K(\tau, t, y(\tau, t)) \mathrm{d} \tau
$$

with

$$
\begin{aligned}
K(x, t, y(x, t))= & -(\mu(x, t) p(x, t))^{-1}\left[\left(\mu(x, t) q(x, t)-(\mu q)_{x}(x, t)\right) y(x, t)\right. \\
& \left.+\left(h(c, t)-\int_{c}^{x} \mu(\tau, t) f(\tau, t, \gamma(\tau, t)) \mathrm{d} \tau\right)\right]
\end{aligned}
$$

and

$$
h(c, t)=-\left[\mu(c, t) p(x, t) \gamma_{x}(c, t)-(\mu p)_{x}(c, t) \gamma(c, t)+\mu(c, t) q(c, t) \gamma(c, t)\right]
$$

has the required properties. This completes the proof. $\square$

Remark 2.4. In 2009, Jung [7]proved the Hyers-Ulam stability of linear partial differential equation of the first order of the following form:

$$
a \gamma_{x}(x, t)+b y_{t}(x, t)+g(x) y(x, t)+h(x)=0
$$

for all $a \geq 0$ and $b>0$.

Now, we consider the generalization of this equation as follows:

$$
a y_{x}(x, t)+b y_{t}(x, t)=f(x, t, y(x, t))
$$

for all $a, b \in \mathbb{R}$ with $a \neq 0$ and $b \neq 0$. Let $\zeta$ and $\eta$ be defined by

$$
\zeta=x-\frac{a}{b} t, \quad \eta=\frac{1}{b} t
$$

If we define $\tilde{\gamma}(\zeta, \eta)=\gamma(\zeta+a \eta, b \eta)=\gamma(x, t)$, then, by (2.13), we have

$$
\begin{gathered}
\gamma_{x}(x, t)=\tilde{y}_{\zeta}(\zeta, \eta) \frac{\partial \zeta}{\partial x}+\widetilde{\gamma}(\zeta, \eta) \frac{\partial \eta}{\partial x^{\prime}} \\
y_{t}(x, t)=\tilde{y}_{\zeta}(\zeta, \eta) \frac{\partial \zeta}{\partial t}+\tilde{y}_{\eta}(\zeta, \eta) \frac{\partial \eta}{\partial t}=-\frac{a}{b} \widetilde{y}_{\zeta}(\zeta, \eta)+\frac{1}{b} \widetilde{y}_{\eta}(\zeta, \eta) .
\end{gathered}
$$

Thus, we see that $a y_{x}(x, t)+b y_{t}(x, t)=\tilde{y}_{\eta}(\zeta, \eta)$, and so we can rewrite the equation (2.12) as follows: 


$$
\tilde{y}_{\eta}(\zeta, \eta)=\tilde{f}\left(\zeta, \eta, \tilde{y}_{\eta}(\zeta, \eta)\right)
$$

Now, we can use Theorem 2.1 for the generalized Hyers-Ulam stability of (2.14).

We consider the mixed type second order nonlinear partial differential equation:

$$
\begin{aligned}
& p(x, t) y_{x t}(x, t)+q(x, t) y_{t}(x, t)+p_{t}(x, t) y_{x}(x, t)-p_{x}(x, t) y_{t}(x, t) \\
= & f(x, t, y(x, t)) .
\end{aligned}
$$

Now, we prove the Hyers-Ulam-Rassias stability of (2.15) under the condition:

$$
p_{x t}(x, t)=q_{t}(x, t)
$$

Theorem 2.5. Let $c \in I p, q: I \times I \rightarrow \mathbb{R}$ be continuous functions with $p(x, t) \neq 0$ for all $x, t \in I, \phi: I \times I \rightarrow(0, \infty)$ be a continuous function, $L: I \times I \rightarrow[1, \infty)$ be an integrable function, and $f: I \times I \times \mathbb{R} \rightarrow \mathbb{R}$ be a continuous function. Assume that there exists $0<\beta<1$ such that

$$
\begin{gathered}
\int_{c}^{x} L(\tau, t) \varphi(\tau, t) \mathrm{d} \tau \leq \beta \varphi(x, t) ; \\
h(x, c)=-\left[p(x, c) y_{x}(x, c)-p_{x}(x, c) \gamma(x, c)+q(x, c) \gamma(x, c)\right] ; \\
K(x, t, \gamma(x, t)) \\
=-(p(x, t))^{-1}\left[\left(p_{x}(x, t)-q(x, t)\right) y(x, t)+h(x, c)-\int_{c}^{t} f(x, \tau, \gamma(x, \tau) \mathrm{d} \tau]\right.
\end{gathered}
$$

and

$$
|K(x, t, u(x, t))-K(x, t, v(x, t))| \leq L(x, t)|u(x, t)-v(x, t)|
$$

for all c, $x, t \in I$ and $h, y, u, v \in C(I \times I)$. Let $y: I \times I \rightarrow \mathbb{R}$ be a function such that

$$
\begin{aligned}
& \left|p(x, t) y_{x t}(x, t)+q(x, t) y_{t}(x, t)+p_{t}(x, t) y_{x}(x, t)-p_{x}(x, t) y_{t}(x, t)-f(x, t, y(x, t))\right| \\
\leq & \varphi(x, t)
\end{aligned}
$$

for all $x, t L I$ and the condition (2.16) holds. Then, there exists a unique solution $y_{0}$ : $I \times I \rightarrow \mathbb{R}$ of (2.15) such that

$$
\left|\gamma(x, t)-y_{0}(x, t)\right| \leq \frac{\beta}{1-\beta} \varphi(x, t) .
$$

Proof. By (2.17) and (2.16), we see that

$$
\begin{aligned}
& \left|p(x, t) y_{x t}(x, t)+q(x, t) y_{t}(x, t)+p_{t}(x, t) y_{x}(x, t)-p_{x}(x, t) y_{t}(x, t)-f(x, t, y(x, t))\right| \\
= & \mid\left(p(x, t) y_{x}(x, t)-p_{x}(x, t) y(x, t)+q(x, t) y(x, t)\right)_{t} \\
& +\left[p_{x t}(x, t)-q_{t}(x, t)\right] y(x, t)-f(x, t, y(x, t)) \mid \\
= & \left|\left(p(x, t) y_{x}(x, t)-p_{x}(x, t) y(x, t)+q(x, t) y(x, t)\right)_{t}-f(x, t, y(x, t))\right|
\end{aligned}
$$

Thus, we have

$$
\begin{aligned}
& -\varphi(x, t) \\
\leq & \left(p(x, t) y_{x}(x, t)-p_{x}(x, t) y(x, t)+q(x, t) y(x, t)\right)_{t}-f(x, t, y(x, t)) \\
\leq & \varphi(x, t) .
\end{aligned}
$$


It follows from (2.18) that

$$
\begin{aligned}
& \left|p(x, t) y_{x}(x, t)-p_{x}(x, t) y(x, t)+q(x, t) y(x, t)+h(c, t)-\int_{c}^{t} f(x, \tau, \gamma(x, \tau)) \mathrm{d} \tau\right| \\
= & |p(x, t)|^{-1} \mid y_{x}(x, t)+(p(x, t))^{-1}\left(\left(q(x, t)-p_{x}(x, t)\right) y(x, t)+h(c, t)\right. \\
& \left.-\int_{c}^{t} f(x, \tau, y(x, \tau)) \mathrm{d} \tau\right) \mid \\
\leq & \int_{c}^{t} \varphi(x, \tau) \mathrm{d} \tau,
\end{aligned}
$$

where

$$
h(x, c)=-\left[p(x, c) y_{x}(x, c)-p_{x}(x, c) y(x, c)+q(x, c) y(x, c)\right] .
$$

From (2.19), we obtain

$$
\begin{aligned}
& \left|y_{x}(x, t)+(p(x, t))^{-1}\left(\left(q(x, t)-p_{x}(x, t)\right) y(x, t)+h(c, t)-\int_{c}^{t} f(x, \tau, y(x, \tau)) \mathrm{d} \tau\right)\right| \\
\leq & |p(x, t)|^{-1} \int_{c}^{t} \varphi(x, \tau) \mathrm{d} \tau .
\end{aligned}
$$

The rest of the proof is similar to that of Theorem 2.2. This completes the proof. $\square$

Remark 2.6. We can define the integrating factor for the equation (2.15) and prove a corollary similar to Corollary 2.3 for Theorem 2.6.

\section{Acknowledgements}

This work was supported by the Korea Research Foundation Grant funded by the Korean Government (KRF-2008-313(00050).

\section{Author details}

${ }^{1}$ Department of Mathematics, Semnan University, P.O. Box 35195-363, Semnan, Iran ${ }^{2}$ Research Group of Nonlinear Analysis and Applications (RGNAA), Semnan, Iran ${ }^{3}$ Centre of Excellence in Nonlinear Analysis and Applications (CENAA), Semnan University, Iran ${ }^{4}$ Department of Mathematics Education and the Rins, Gyeongsang National University, Chinju 660-701, Korea ${ }^{5}$ Department of Mathematics, Iran University of Science and Technology, Narmak, Tehran, Iran ${ }^{6}$ Graduate Center, Payame Noor University, Shahnaz Alley, Haj Mahmood Norian Street, Tehran, Iran

${ }^{7}$ Tabriz College of Technology, P.O. Box 51745-135, Tabriz, Iran

\section{Authors' contributions}

$A B$ carried out the molecular genetic studies, participated in the sequence alignment and drafted the manuscript. JY carried out the immunoassays. MT participated in the sequence alignment. ES participated in the design of the study and performed the statistical analysis. FG conceived of the study and participated in its design and coordination. All authors read and approved the final manuscript.

\section{Competing interests}

The authors declare that they have no competing interests.

Received: 8 December 2010 Accepted: 7 October 2011 Published: 7 October 2011

\section{References}

1. Alsina, C, Ger, R: On some inequalities and stability results related to the exponential function. J Inequal Appl. 2, 373-380 (1998). doi:10.1155/S102558349800023X

2. Takahasi, SE, Miura, T, Miyajima, S: On the Hyers-Ulam stability of Banach space-valued differential equation $y^{\prime}=\lambda y$, Bull. Korean Math Soc. 39, 309-315 (2002). doi:10.4134/BKMS.2002.39.2.309

3. Jung, SM: Hyers-Ulam stability of linear differential equations of firsty order III. J Math Anal Appl. 311, $139-146$ (2005). doi:10.1016/j.jmaa.2005.02.025 
4. Jung, SM: Hyers-Ulam stability of linear differential equations of firsty order II. Appl Math Lett. 19, 854-858 (2006). doi:10.1016/j.aml.2005.11.004

5. Jung, SM: A fixed point approach to the stability of differential equations $y^{\prime}=F(x, y)$. Bull Malays Math Sci Soc. 33, 47-56 (2010)

6. Jung, SM, Min, S: On approximate Eular differential equations. Abstr Appl Anal 2009 (2009). (Article ID 537963), 8 pages

7. Jung, SM: Hyers-Ulam stability of linear partial differential equations of first order. Appl Math Lett. 22, 70-74 (2009). doi:10.1016/j.aml.2008.02.006

8. Li, Y, Shen, Y: Hyers-Ulam stability of linear differential equations of scecond order. Appl Math Lett. 23, 306-309 (2010). doi:10.1016/j.aml.2009.09.020

9. Baker, J: The stability of the cosin equation. Proc Amer Math Soc. 80, 411-416 (1980). doi:10.1090/S0002-9939-19800580995-3

10. Cholewa, PW: Remarks on the stability of functional equations. Aequat Math. 27, 76-86 (1984). doi:10.1007/BF02192660

11. Czerwik, S: On the stability of the quadratic mapping in normed spaces. Abh Math Sem Univ Hamburg. 62, 59-64 (1992). doi:10.1007/BF02941618

12. Gordji, ME, Karimi, T, Gharetapeh, SK: Approximately n-Jordan homomorphisms on Banach algebras. J Ineq Appl 2009 (2009). (Article ID 870843), 8 pages

13. Eshaghi Hordji, M, Ghaemi, MB, Kaboli Gharetapeh, S, Shams, S, Ebadian, A: On the stability of J*-derivations. J Geom Phy. 60, 454-459 (2010). doi:10.1016/j.geomphys.2009.11.004

14. Găvruta, P, Găvruta, L: A new method for the generalized Hyers-Ulam-Rassias stability. Intern J Nonlinear Anal Appl. 1, $11-18(2010)$

15. Gordji, ME, Zolfaghari, S, Rassias, JM, Savadkouhi, MB: Solution and stability of a mMixed type cubic and qqartic functional equation in quasi-Banach spaces. Abstr Appl Anal 2009 (2009). (Article ID 417473), 14 pages

16. Hyers, DH: On the stability of the linear functional equation. Proc Natl Acad Sci USA. 27, 222-224 (1941). doi:10.1073/ pnas.27.4.222

17. Hyers, DH, Isac, G, Rassias, TM: Stability of Functional Equations in Several Variables. Birkhausar, Basel (1998)

18. Khodaei, H, Rassias, TM: Approximately generalized additive functions in several variable. Intern J Nonlinear Anal Appl. 1, 22-41 (2019)

19. Rassias, TM: On the stability of the linear mapping in Banach spaces. Proc Amer Math Soc. 72, 297-300 (1978). doi:10.1090/S0002-9939-1978-0507327-1

20. Ulam, SM: Problems in Modern Mathematics, Chapter VI, Science ed. Wiley, New York (1940)

doi:10.1186/1029-242X-2011-81

Cite this article as: Gordji et al:: Stability of the second order partial differential equations. Journal of Inequalities and Applications 2011 2011:81.

\section{Submit your manuscript to a SpringerOpen ${ }^{\circ}$} journal and benefit from:

Convenient online submission

- Rigorous peer review

- Immediate publication on acceptance

- Open access: articles freely available online

- High visibility within the field

- Retaining the copyright to your article

Submit your next manuscript at $\boldsymbol{s p r i n g e r o p e n . c o m ~}$ 